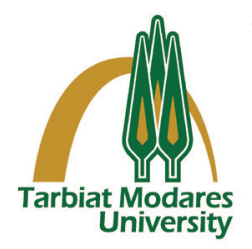

\title{
In Vitro Evaluation of Antimicrobial Properties of Some Newly Synthesized S-Triazole Thioglycosides
}

\section{A R T I C L E I N F O}

Article Type

Original Research

Authors

Razieh Firoozi, $M S c^{1}$

Karim Akbari Dilmaghani, $P h D^{1^{*}}$

Zahra Dono Ghezelbash, $P h D^{1}$

Yasin SarveAhrabi, $P h D^{2}$

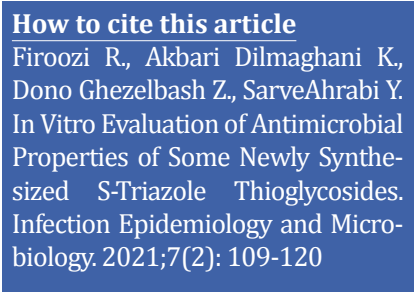

${ }^{1}$ Department of Organic Chemistry, Faculty of Chemistry, Urmia University, Urmia, Iran ${ }^{2}$ Department of Biology, Central Tehran Branch, Islamic Azad University, Tehran, Iran

\section{* Correspondence \\ Address: EDepartment of Organic Chemistry, Faculty of Chemistry, Ur- mia University, Urmia, Iran. k.adilmaghani@urmia.ac.ir}

\begin{abstract}
A B S T R A C T
Backgrounds: Nowadays, the need for replacement of new drug structures is felt more than ever due to the spread of microbial resistance. S-triazoles are significant five-membered heterocyclic scaffolds due to their wide range of biological activities.

Materials \& Methods: A new series of Schiff bases (5a-f) were synthesized by the reaction of 4-amino-S-triazoles (3a-c) with furan and benzaldehyde $4(d-e)$. Then a novel series of triazole thioglycosides (7a-f) were synthesized by the reaction of Schiff bases (5a-f) and $\mathrm{T}-0$-acetyle- $\alpha$-D-glucopyranosyle-Br in the presence of potassium carbonate as a weak base in acetone. The structure of the products was confirmed by FT-IR, H-NMR, and C-NMR assays. The antimicrobial properties of the newly synthesized compounds were studied against four bacterial strains, including Bacillus cereus, Staphylococcus aureus, Pseudomonas aeruginosa, and Escherichia coli, and two fungal strains, including Aspergillus niger and Candida albicans.

Findings: The synthesized compounds exhibited better antifungal activity than antibacterial activity, espetially 7d. Among all the compounds, the compound $7 \mathrm{~d}$ was found to have the highest activity against $C$. albicans with $\mathrm{IZ}=18 \pm 0.7 \mathrm{~mm}, \mathrm{MIC}=250 \mathrm{mg} / \mathrm{mL}$, and $\mathrm{MFC}=250 \mathrm{mg} / \mathrm{mL}$.

Conclusion: The present study results indicated that compounds containing S-triazole had the potential to be used in a wide variety of new antifungal formulations.
\end{abstract}

Keywords: Triazoles, Candida albicans, Drug resistance.

\section{CITATION LINKS}

[1] Willyard C. The drug-resistant bacteria ... [2] Morris S, Cerceo E. Trends, epidemiology, and ... [3] Sultan I, Rahman S, Jan AT, Siddiqui MT, Mondal ... [4] Maddila S, Momin M, Gorle S, Palakondu L, Jonnalagadda ... [5] Sangshetti JN, Lokwani DK, Sarkate AP, Shinde DB. Synthesis ... [6] Ragenoviќ KC, Dimova V, Kakurinov V, Gabor DM, Buzarovska A. Synthesis and ... [7] Mathew V, Keshavayya J, Vaidya VP, Giles D. Studies on synthesis and ... [8] Altıntop MD, Kaplancıklı ZA, Turan-Zitouni G, Özdemir A, İșcan G, Akalın G, et al. Synthsis and ... [9] Mathew V, Giles D, Keshavayya J, Vaidya VP. Studies on synthesis and ... [10] Ahmadi F, Rezayan Ghayahbashi M, Sharifzadeh M, Alipoiur E, Nasser Ostad S, Vosooghi M, et al. Synthesis and ... [11] Kaur R, Ranjan Dwivedi A, Kumar B, Kumar V. Recent ... [12] Ronad PM, Noolvi MN, Sapkal S, Dharbhamulla S, Maddi VS. Synthesis ... [13] Dilmaghani KA, Jazani NH. Nasuhi Pur F, Shokoufeh N, Ghadiri F, Mirfakhraee ... [14] Gupta D, Jain DK. Synthesis, antifungal, and antibacterial ... [15] Dilmaghani KA, Nasuhi Pur F, Hataminezhad M. Synthesis and ... [16] Dhapalapur MG, Sabnis SS, Deliwala CV. Potential anticancer agents. II. Schiff ... [17] Khalil NS. Efficient synthesis, structure, and antimicrobial activity of ... [18] Tiwari J, Saquib M, Singh S, Tufail F, Singh J, Singh J. Catalyst ... [19] Perrin DD, Armarego WL, Perrin DR. Purification of ... [20] Beyer H, Kroger CF, Bussel G. 1, 2, 4-Triazoles. I. The reaction ... [21] de Mora SJ, Fowler SW, Cassi R, Tolosa I. Assessment ... [22] Furniss BS. Text book of ... [23] Dilmaghani KA, Pur FN, Jazani NH, Alavi A, Niknam Z, Mirfakhraee ... [24] Zarrabi N, souldozi A, SarveAhrabi Y. In vitro evaluation of ... [25] Senda Y, Imaizumi S. Carbon-13 pulse Fourier transform ... [26] Kelarev VI, Shvekhgeimer GA, Lunin AF. Synthesis of 2-amino ... [27] Gopinathan S, Pardhy SA, Budhkar AP, Gopinathan C. Ruthenium (II) complexes ... [28] Ghadiri H, Vaez H, Khosravi S, Soleymani E. The antibiotic ... [29] Micoli F, Bagnoli F, Rappuoli R, Serruto D. The role of ... [30] El Ashry ES, Elshatanofy MM, Badawy ME, Kandeel KM, Elhady OM, Abdel-Sayed MA. Synthesis ... [31] Revanasiddappa BC, Kumar MV, Nayak P, Ali AR, Jisha MS. Synthesis ... [32] Godeau C, Morin-Crini N, Staelens JN, Martel B, Rocchi S, Chanet G, et al. Adsorption ...

\section{Article History}

Received: March 022021

Accepted: May 03,2021

Published: May 20,2021

Copyright(C 2021, TMU Press. This open-access article is published under the terms of the Creative Commons Attribution-NonCommercial 4.0 International License which permits Share (copy and redistribute the material in any medium or format) and Adapt (remix, transform, and build upon the material) under the Attribution-NonCommercial terms. 


\section{Introduction}

Nowadays, there is a long list of drugresistant microbes, including sulfonamideresistant, penicillin-resistant, methicillinresistant, macrolide - resistant, multicellular, and vancomycin-resistant microbes [1]. As a result, treatment of drug-resistant bacterial diseases requires large amounts of drugs and sedative treatments for a long time, which eventually leads to incremented mortality. In addition, the widespread and aimless use of powerful antibiotics in recent decades has led to the emergence of a large number of microbial resistances, which are currently considered as one of the most important concerns and serious threats to global public health ${ }^{[2]}$. In general, the classification of antibiotics is based on their mechanism of action, for example, interference with cell wall synthesis, interference with the cell proliferation cycle, and disruption of the cell cycle ${ }^{[3]}$. Considering the advances in recent decades, especially in the science of synthesis of pharmaceutical structures, it seems the main strategy against microbial resistance is to develop new drug structures. Nowadays, S-triazole core is considered as a significant moiety in the design and synthesis of bioactive compounds possessing many biological properties, including anti-microbial [45], antibacterial ${ }^{[6]}$, antifungal [7], anti-HIV [8], anti-inflammatory [9], analgesic [10] and anticancer activities ${ }^{[11]}$. Fungal and bacterial infections have become a major challenge and an important cause of fatality. Based on these observations, S-triazole derivatives may be considered as possible candidates for use as safe antimicrobial agents. Thus, there is a need to prospect these pharmacophores for the extension of modern molecules with several activities. [12]. Schiff bases have attracted much interest due to their synthetic availability along with antibacterial [13-15] and antitumor [16] properties. The synthesis and verification of the biological activity of S-triazole glycosides have been motivated by the detection of Ribavirin ${ }^{[17]}$. It has been reported that coupling of carbohydrate derivatives to the $\mathrm{S}$-triazole nucleus through a thioglycosidic linkage enhances its biological activities. Some novel S- $\beta$-Dglucosides of 5-aryl-S-triazole-3-thiones derivatives have been shown to exhibite biological activities [18].

Objectives: Due to mentioned findings, this study aimed to design potent antibacterial agents with S-triazole moieties, to synthesis some new substituted thioglycosides via the reaction of $\alpha$-D- aceto- bromo-glucose with 4- amino- 5- alkyl- 4H- S-triazole- 3thiol Schiff bases (5a-f), and to investigate the newly synthesized hybrid compounds (7a-f) in terms of biological activities such as antibacterial and antifungal effects.

\section{Materials and Methods}

This research was conducted in the chemical laboratories of Urmia University in collaboration with the Microbiology Laboratory of the Islamic Azad University, Tehran Branch in 2020. Starting materials, solvents, and culture environments (nutrient agar/broth, sabouraud dextroseand agar/ broth) were obtained from Merck, Germany and used without any more filtration. FT-IR, as well as H-NMR and C-NMR spectra were recorded on Thermo Nicolet Nexus-670 and Bruker Avance-300MHz spectrometers, respectively. Thin layer chromatography (TLC) analyses were carried out on silica gel plates. All chemicals were purchased from Merck (Germany) and used according to standard procedures. Newly distilled solvents were used throughout the study, and anhydrous solvents were dried according to the method reported by Perrin and Armarego (1988) ${ }^{[19]}$. All bacterial and fungal strains were prepared f rom the Iranian Industrial Microorganisms Collection Center (Lyophilized). Microbio logical tests were performed using a Memmert- INC153T2T3 


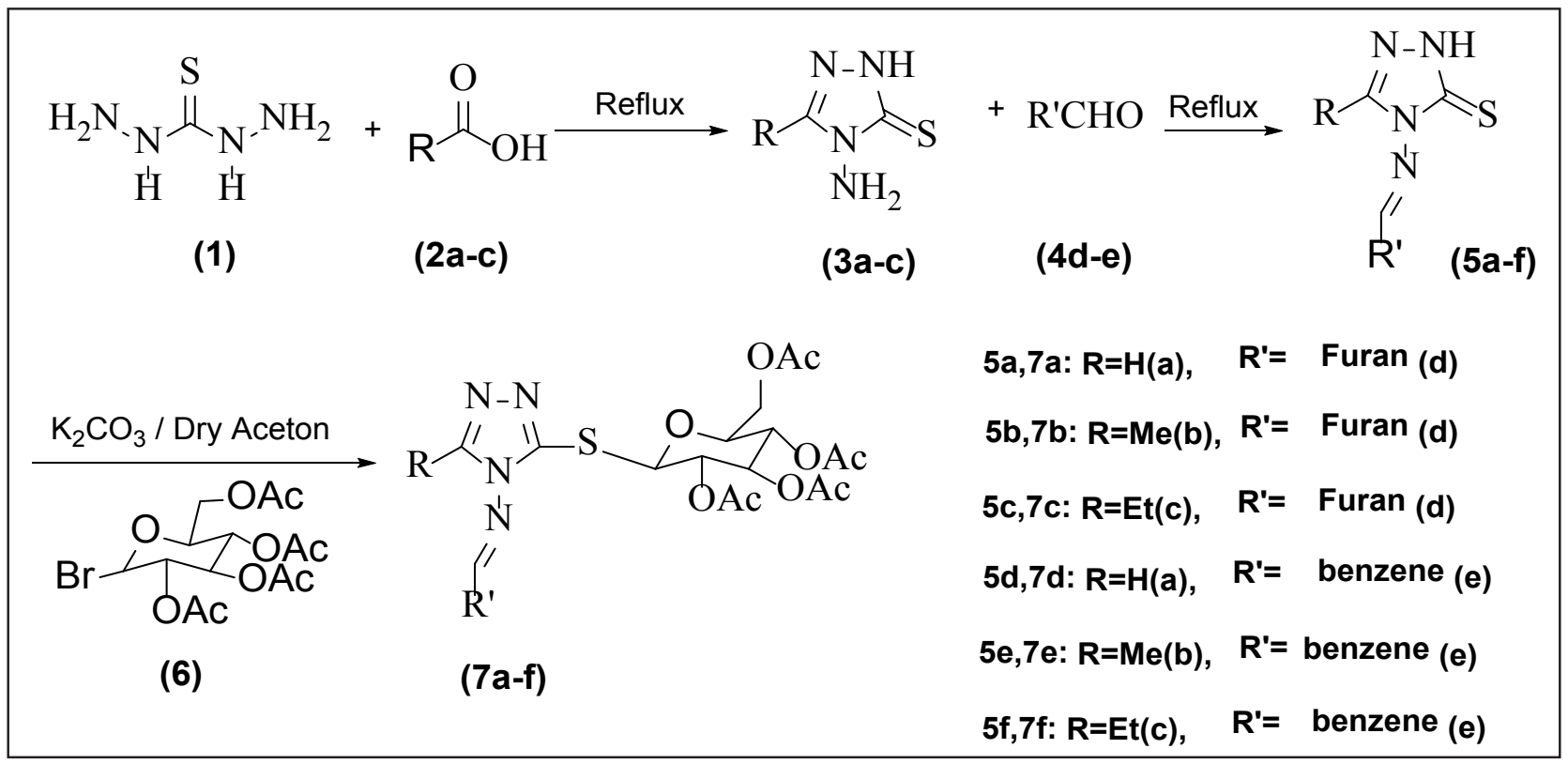

Scheme1) Synthesis of S- $\beta$-Dglycosides-4-arylideneamino-5-alkyl-S-triazole-3-thione

incubator.

Chemistry: 1. General synthesis of 4amino- 5- alkyl- 4H- S-triazole- 3- thiol (3a-c): At first, carboxylic acid (0.01 mol) was added to thiocarbohydrazide $(0.01 \mathrm{~mol})$ at $150{ }^{\circ} \mathrm{C}$ for $15 \mathrm{~min}$ and then refluxed for $40 \mathrm{~min}$. After cooling, the obtained product was treated with a sodium bicarbonate solution. The compound was then washed with water and collected by filtration. The solid product was recrystallized from instiled water (Scheme1) ${ }^{[20] .}$

2. General synthesis of Schiff bases of (E)5- alkyl- 4- ((benzyliden/ furfuraldyn) amino) Alkyl-2, 3-dihydro- 4H-S-triazole3-thione (5a-f): At first, substituted amino mercapto triazole $(0.1 \mathrm{~mol})$ (3ac) in ethanol (1 $\mathrm{mL}$ ) was added to aryl carboxaldehyde ( $4 \mathrm{~d}-\mathrm{e})$ ( $0.1 \mathrm{~mol})$. Then sulfuric acid was added to the previous solution and heated for 6 hrs.

The precipitated solid was filtered off and recrystallized in ethanol (Scheme1) ${ }^{[21]}$.

3. General synthesis of $2,3,4,6$-tetra- 0 acetyl- $\alpha$ - D-glucopyranosyl bromid (6): It was also prepared according to the literature procedure (Scheme1) ${ }^{[22]}$. 4. General synthesis of 3-S- $\beta$ - D-glucosides-
4- arylideneamino- 5- alkyl- S-triazoles (7a-f): At first, compound (5a-f) (1 mmol) was added to potassium carbonate (1 mmol ) in dry acetone (25 $\mathrm{mL})$ along with 2 drops of Dimethylformamide and stirred for $1 \mathrm{hrs}$, then glycosyl bromid (1.2 mmol) was added, stirred for $12 \mathrm{hrs}$, and heated under reflux for 2-4 hrs. After cooling, the mixture was filtered, then the precipitate of the compound was submitted to column chromatography (Scheme1) ${ }^{[23]}$.

Preparation of compound concentrations: Dimethyl sulfoxide (99\%) (DMSO) was used to dissolve all compounds. Initially, a concentration of $0.5 \mathrm{mg} / \mathrm{mL}$ was prepared from the powders of synthesized compounds (1:9 ratios). Afterwards, they were kept at $-18^{\circ} \mathrm{C}$ in sterile test tubes until the tests were performed.

Antimicrobial Activity: Agar well diffusion (inhibition zone), MIC (minimum inhibitory concentration), and MBC (minimum bactericidal concentration)/ MFC (minimum fungicidal concentration) methods were used to evaluated the antibacterial and antifungal properties of the newly synthesized compounds.

Preparation of bacterial and fungal suspension: The lipophilic ampoules containing Bacillus cereus, Staphylococcus 
Compound(7a): 4- (furfuraldehyde amino) -2 -yl -3- (2, 3, 4, 6-tetra-O- acetyl- $\beta$-D-gluco pyranosyl Sulfonyl) -S-triazole (light yellow crystals), (yield\% $\%$ 73) ( $0.70 \mathrm{~g}),(\mathrm{m} . \mathrm{p}: 160-163)$

\begin{tabular}{|c|c|}
\hline \multirow{3}{*}{$\mathrm{H}_{\mathrm{N}}^{\mathrm{N}-\mathrm{N}}{ }_{\mathrm{OAc}}^{\mathrm{OAAc}}$} & $\begin{array}{l}\left.\text { FT-IR (KBr, v cm} \mathbf{~ c m}^{-1}\right): 2975(\mathrm{C}-\mathrm{H}), 1749(\mathrm{C}=0), 1620(\mathrm{HC}=\mathrm{N}) \text {, } \\
1376\left(\mathrm{CH}_{3}\right), 1232,1042(\mathrm{C}-\mathrm{O})\end{array}$ \\
\hline & $\begin{array}{l}{ }^{1} \mathrm{H}-\mathrm{NMR}\left(\mathbf{3 0 0} \mathbf{M H z}, \mathrm{CDCl}_{3}\right): \delta(\mathrm{ppm}), 1.93(\mathrm{~s}, 3 \mathrm{H}, \mathrm{OAc}), 2.04 \\
(\mathrm{~s}, 3 \mathrm{H}, \mathrm{OAc}), 2.06(\mathrm{~s}, 3 \mathrm{H}, \mathrm{OAc}), 2.09(\mathrm{~s}, 3 \mathrm{H}, \mathrm{OAc}), 3.98-4.01(\mathrm{~m}, \\
1 \mathrm{H}, \mathrm{H}-5), 4.14-4.19(\mathrm{~m}, 1 \mathrm{H}, \mathrm{H}-6 \mathrm{a}), 4.28-4.33(\mathrm{~m}, 1 \mathrm{H}, \mathrm{H}-6 \mathrm{~b}), \\
5.25(\mathrm{t}, \mathrm{J}=9 \mathrm{~Hz}, 1 \mathrm{H}, \mathrm{H}-2), 5.42(\mathrm{t}, \mathrm{J}=9.6 \mathrm{~Hz}, 1 \mathrm{H}, \mathrm{H}-4) 5.76(\mathrm{t}, \mathrm{J}=9 \\
\mathrm{Hz}, 1 \mathrm{H}, \mathrm{H}-3), 6.19(\mathrm{~d}, \mathrm{~J}=9.3 \mathrm{~Hz}, 1 \mathrm{H}, \mathrm{H}-1), 6.60-6.61(\mathrm{~d}, \mathrm{~J}=1.8 \\
\mathrm{Hz}, 1 \mathrm{H}, \text { Furyl), } 7.06-7.07(\mathrm{~d}, \mathrm{~J}=3.3 \mathrm{~Hz}, 1 \mathrm{H}, \text { Furyl), } 7.68(\mathrm{~s}, 1 \mathrm{H}, \\
\text { Furyl), } 8.07(\mathrm{~s}, 1 \mathrm{H}, \mathrm{H}-\mathrm{C} \text { triazole), } 10.48(\mathrm{~s}, 1 \mathrm{H}, \mathrm{HC}=\mathrm{N}) .\end{array}$ \\
\hline & $\begin{array}{l}{ }^{13} \mathrm{C}-\mathrm{NMR}\left(\mathbf{7 5} \mathbf{M H z}, \mathrm{CDCl}_{3}\right): \delta(\mathrm{ppm}), 20.43,67.09,68.39,73.77 \text {, } \\
75.39,76.62,80.31,112.6,118.9,141.54,145.77,163.72, \\
\text { 168.85, 169.35, 170.14, 170.60. Found: C, 48.12; H, 4.51; N, } \\
10.88 ; \mathrm{S}, 6.25 \% .\end{array}$ \\
\hline \multicolumn{2}{|c|}{$\begin{array}{l}\text { Compound(7b): 4- (furfuraldehyde amino)- 5- methyl- 2- yl- 3- (2, 3, 4, 6-tetra-0-acetyl- } \beta \text {-D-gluco pyranosyl- } \\
\text { Sulfonyl) -S-triazole (light yellow crystals), (yield } \%=79)(0.81 \mathrm{~g}),\left(\mathrm{m} . \mathrm{p}: 130-132^{\circ} \mathrm{C}\right)\end{array}$} \\
\hline \multirow{3}{*}{$\overbrace{\substack{1 \\
N}}^{\mathrm{N}-\mathrm{N}}$} & $\begin{array}{l}\text { FT-IR (KBr, v cm c-1): } 2961(\mathrm{C}-\mathrm{H}), 1751(\mathrm{C}=0), 1607(\mathrm{HC}=\mathrm{N}) \text {, } \\
1376\left(\mathrm{CH}_{3}\right), 1235,1044(\mathrm{C}-\mathrm{O})\end{array}$ \\
\hline & $\begin{array}{l}{ }^{1} \mathrm{H}-\mathrm{NMR}\left(\mathbf{3 0 0} \mathbf{M H z} \mathrm{CDCl}_{3}\right): \delta(\mathrm{ppm}), 1.93(\mathrm{~s}, 3 \mathrm{H}, \mathrm{OAc}), 2.03(\mathrm{~s}, \\
3 \mathrm{H}, \mathrm{OAc}), 2.06(\mathrm{~s}, 3 \mathrm{H}, \mathrm{OAc}), 2.09(\mathrm{~s}, 3 \mathrm{H}, \mathrm{OAc}), 2.45\left(\mathrm{~s}, 3 \mathrm{H}, \mathrm{CH}_{3}\right) \text {, } \\
3.96-3.99(\mathrm{~m}, 1 \mathrm{H}, \mathrm{H}-5), 4.13-4.18(\mathrm{~m}, 1 \mathrm{H}, \mathrm{H}-6 \mathrm{a}), 4.28-4.34 \\
(\mathrm{~m}, 1 \mathrm{H}, \mathrm{H}-6 \mathrm{~b}), 5.25(\mathrm{t}, \mathrm{J}=9.6 \mathrm{~Hz}, 1 \mathrm{H}, \mathrm{H}-2), 5.41(\mathrm{t}, \mathrm{J}=9.3 \mathrm{~Hz}, 1 \mathrm{H}, \\
\mathrm{H}-4) 5.73(\mathrm{t}, \mathrm{J}=9.3 \mathrm{~Hz}, 1 \mathrm{H}, \mathrm{H}-3), 6.207(\mathrm{~d}, \mathrm{~J}=9.3 \mathrm{~Hz}, 1 \mathrm{H}, \mathrm{H}-1) \text {, } \\
6.60-6.61(\mathrm{~d}, \mathrm{~J}=1.5 \mathrm{~Hz}, 1 \mathrm{H}, \text { Furyl), } 7.06-7.07(\mathrm{~d}, \mathrm{~J}=3 \mathrm{~Hz}, 1 \mathrm{H}, \\
\text { Furyl), } 7.68(\mathrm{~s}, 1 \mathrm{H}, \text { Furyl), } 10.32(\mathrm{~s}, 1 \mathrm{H}, \mathrm{HC}=\mathrm{N})\end{array}$ \\
\hline & $\begin{array}{l}\left.{ }^{13} \text { C-NMR (75 MHz, } \text { CDCl }_{3}\right): \delta(\mathrm{ppm}), 11.13,20.33,61.55,66.34 \text {, } \\
68.49,73.63,76.63,80.01,111.52,11363,145.65,149.17 \\
\text { 163.79, 168.89, 169.33, 170.15, 170.20. Found: C, 49.11; H, } \\
\text { 4.77; N, 10.48; S, } 5.87 \% .\end{array}$ \\
\hline \multicolumn{2}{|c|}{$\begin{array}{l}\text { Compound(7c): 4- (furfuraldehyde amino)-5- etyl- 2- yl- 3- }(2,3,4 \text {, 6-tetra- } 0 \text { - acetyl- } \beta \text { - D-gluco pyranosyl } \\
\text { Sulfonyl) -S-triazole (light brown crystals), (yield } \%=78)(0.72 \mathrm{~g}),\left(\mathrm{m} . \mathrm{p}: 87-89^{\circ} \mathrm{C}\right)\end{array}$} \\
\hline \multirow{3}{*}{$\overbrace{\substack{\mathrm{N} \\
\mathrm{N}}}^{\mathrm{N}-\mathrm{N}}$} & $\begin{array}{l}\left.\text { FT-IR (KBr, v cm }{ }^{-1}\right): 2951(\mathrm{C}-\mathrm{H}), 1753(\mathrm{C}=0), 1608(\mathrm{HC}=\mathrm{N}) \\
1373\left(\mathrm{CH}_{3}\right), 1230,1043(\mathrm{C}-\mathrm{O})\end{array}$ \\
\hline & 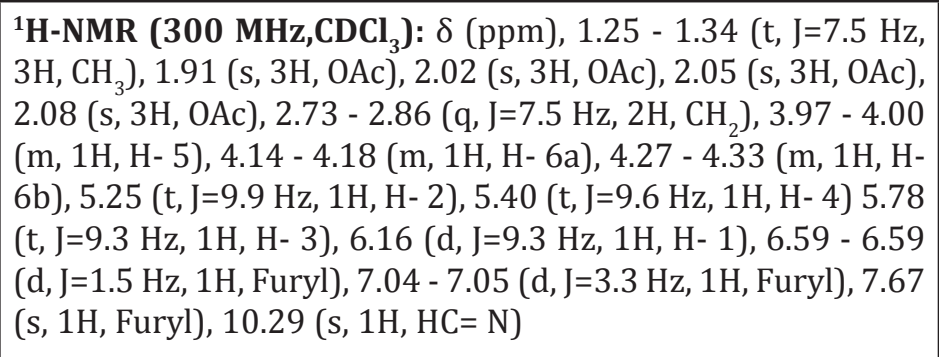 \\
\hline & $\begin{array}{l}{ }^{13} \mathrm{C}-\mathrm{NMR}\left(\mathbf{7 5} \mathbf{M H z}, \mathbf{C D C l}_{3}\right): \delta(\mathrm{ppm}), 20.22,62.65,66.42,67.17 \\
73.59,76.61,80.85,153.07,153.02,150.08,111.63,113.76 \text {, } \\
\text { 148.52, 149.16, 163.93. Found: C, 50.19; H, 5.21; N, 10.01; S, } \\
5.70 \% .\end{array}$ \\
\hline
\end{tabular}

Figure 1) Structural and spectral information of newly synthesized derivatives 


\begin{tabular}{|c|c|}
\hline \multicolumn{2}{|c|}{$\begin{array}{l}\text { Compound(7d): } 4 \text { - (benzylidene amino)- } 2-\text { yl- } 3-(2,3,4,6 \text { - tetra- } 0 \text { - acetyl- } \beta \text { - D- gluco pyranosyl Sulfonyl) } \\
\text {-S-triazole (white crystals), (yield } \%=71)(0.61 \mathrm{~g}),\left(\text { m.p: } 162-164{ }^{\circ} \mathrm{C}\right)\end{array}$} \\
\hline \multirow{3}{*}{$\mathrm{N}_{\mathrm{N}}^{\mathrm{N}}{ }_{\text {OAc }}^{\mathrm{N}} \mathrm{OAc}$} & $\begin{array}{l}\text { FT-IR (KBr, v cm-1 }): 2937(\mathrm{C}-\mathrm{H}), 1748(\mathrm{C}=0), 1368\left(\mathrm{CH}_{3}\right) \text {, } \\
\text { 1045, } 1233(\mathrm{C}-\mathrm{O})\end{array}$ \\
\hline & $\begin{array}{l}{ }^{1} \mathbf{H}-\mathbf{N M R}\left(300 \mathbf{M H z} \mathbf{C D C l}_{3}\right): \delta(\mathrm{ppm}), 1.95(\mathrm{~s}, 3 \mathrm{H}, \mathrm{OAc}), 2.04 \\
(\mathrm{~s}, 3 \mathrm{H}, \mathrm{OAc}), 2.07(\mathrm{~s}, 3 \mathrm{H}, \mathrm{OAc}), 2.09(\mathrm{~s}, 3 \mathrm{H}, \mathrm{OAc}), 3.98-4.03(\mathrm{~m}, \\
1 \mathrm{H}, \mathrm{H}-5), 4.15-4.19(\mathrm{~m}, 1 \mathrm{H}, \mathrm{H}-6 \mathrm{a}), 4.28-4.34(\mathrm{~m}, 1 \mathrm{H}, \mathrm{H}-6 \mathrm{~b}), \\
5.26(\mathrm{t}, \mathrm{J}=9 \mathrm{~Hz}, 1 \mathrm{H}, \mathrm{H}-2), 5.43(\mathrm{t}, \mathrm{J}=9.6 \mathrm{~Hz}, 1 \mathrm{H}, \mathrm{H}-4) 5.78(\mathrm{t}, \\
\mathrm{J}=9.3 \mathrm{~Hz}, 1 \mathrm{H}, \mathrm{H}-3), 6.23(\mathrm{~d}, \mathrm{~J}=9.3 \mathrm{~Hz}, 1 \mathrm{H}, \mathrm{H}-1), 7.46-7.56(\mathrm{~m}, \\
3 \mathrm{H}, \mathrm{Ar}), 7.84-7.87(\mathrm{~m}, 2 \mathrm{H}, \mathrm{Ar}), 8.07(\mathrm{~s}, 1 \mathrm{H}, \mathrm{H}-\mathrm{C} \text { triazole }), 10.37 \\
(\mathrm{~s}, 1 \mathrm{H}, \mathrm{HC}=\mathrm{N})\end{array}$ \\
\hline & $\begin{array}{l}{ }^{13} \mathrm{C}-\mathrm{NMR}\left(\mathbf{7 5} \mathbf{M H z}_{\mathbf{C}} \mathbf{C D C l}_{3}\right): \delta(\mathrm{ppm}), 20.57,73.67,75.40,77.07, \\
77.49,80.17,80.41,127.74,129.75,129.87,130.12,161.68, \\
168.94,169.39,170.13,170.49 . \text { Found: C, 51.78; H, 4.80; N, } \\
10.58 ; \mathrm{S}, 6.19 \% .\end{array}$ \\
\hline
\end{tabular}

Compound(7e): 4- (benzylidene amino)- 5- methyl- 2- yl- 3- (2, 3, 4, 6-tetra-O-acetyl- $\beta$-D-gluco pyranosyl Sulfonyl) -S-triazole (light yellow crystals), (yield\% = 70), (0.51 g), (m.p: $\left.110-112{ }^{\circ} \mathrm{C}\right)$

\begin{tabular}{|c|c|}
\hline \multirow{3}{*}{$\overbrace{\mathrm{N}_{\mathrm{N}}}^{\mathrm{N}_{-}^{\mathrm{N}}} \underbrace{\mathrm{OAAc}}_{\text {OAc }}$} & $\begin{array}{l}\text { FT-IR (KBr, v cm-1): } 2961(\mathrm{C}-\mathrm{H}), 1752(\mathrm{C}=0), 1605(\mathrm{HC}=\mathrm{N}) \text {, } \\
1370\left(\mathrm{CH}_{3}\right), 1045,1230(\mathrm{C}-\mathrm{O})\end{array}$ \\
\hline & $\begin{array}{l}{ }^{1} \mathrm{H}-\mathrm{NMR}\left(\mathbf{3 0 0} \mathbf{M H z} \mathbf{C D C l}_{3}\right): \delta(\mathrm{ppm}), 1.95(\mathrm{~s}, 3 \mathrm{H}, \mathrm{OAc}), 2.04 \\
(\mathrm{~s}, 3 \mathrm{H}, \mathrm{OAc}), 2.07(\mathrm{~s}, 3 \mathrm{H}, \mathrm{OAc}), 2.10(\mathrm{~s}, 3 \mathrm{H}, \mathrm{OAc}), 2.47(\mathrm{~s}, 3 \mathrm{H}, \\
\left.\mathrm{CH}_{3}\right), 3.97-4.01(\mathrm{~m}, 1 \mathrm{H}, \mathrm{H}-5), 4.14-4.18(\mathrm{~m}, 1 \mathrm{H}, \mathrm{H}-6 \mathrm{a}), 4.27 \\
-4.35(\mathrm{~m}, 1 \mathrm{H}, \mathrm{H}-6 \mathrm{~b}), 5.26(\mathrm{t}, \mathrm{J}=9.6 \mathrm{~Hz}, 1 \mathrm{H}, \mathrm{H}-2), 5.43(\mathrm{t}, \mathrm{J}=9.3 \\
\mathrm{Hz}, 1 \mathrm{H}, \mathrm{H}-4) 5.76(\mathrm{t}, \mathrm{J}=9.3 \mathrm{~Hz}, 1 \mathrm{H}, \mathrm{H}-3), 6.23(\mathrm{~d}, \mathrm{~J}=9.3 \mathrm{~Hz}, 1 \mathrm{H}, \\
\mathrm{H}-1), 7.47-7.56(\mathrm{~m}, 3 \mathrm{H}, \mathrm{Ar}), 7.85-7.88(\mathrm{~m}, 2 \mathrm{H}, \mathrm{Ar}), 10.40(\mathrm{~s}, \\
1 \mathrm{H}, \mathrm{HC}=\mathrm{N})\end{array}$ \\
\hline & $\begin{array}{l}{ }^{13} \mathrm{C}-\mathrm{NMR}\left(\mathbf{7} \mathbf{~ M H z}, \mathbf{C D C l}_{3}\right): \delta(\mathrm{ppm}), 11.27,20.60,66.96,67.12, \\
69.70,76.64,77.07,80.34,127.65,127.93,129.77,130.10, \\
160.56,163.94,168.95,169.34,169.37 . \text { Found: C, 52.65; H, } \\
\text { 5.28; N, 10.10; S, 5.79\%. }\end{array}$ \\
\hline \multicolumn{2}{|c|}{$\begin{array}{l}\text { Compound(7f): 4- (benzylidene amino)- 5- ethyl- 2- yl- 3- }(2,3,4 \text {, 6- tetra-0-acetyl- } \beta \text {-D-gluco pyranosyl } \\
\text { Sulfonyl) -S-triazole (white crystals), (yield } \%=79)(0.61 \mathrm{~g}),\left(\mathrm{m} . \mathrm{p}: 131-133{ }^{\circ} \mathrm{C}\right)\end{array}$} \\
\hline \multirow{3}{*}{ 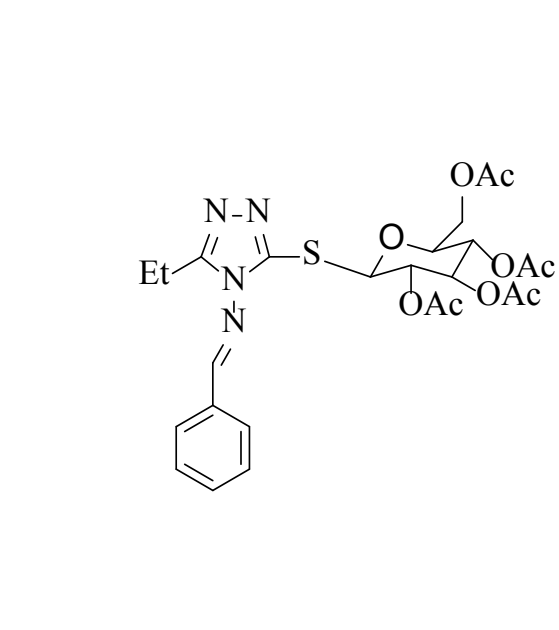 } & $\begin{array}{l}\text { FT-IR (KBr, u cm-1): } 2955(\mathrm{C}-\mathrm{H}), 1756(\mathrm{C}=0), 1593(\mathrm{HC}=\mathrm{N}) \text {, } \\
1369\left(\mathrm{CH}_{3}\right), 1039,1231(\mathrm{C}-\mathrm{O})\end{array}$ \\
\hline & $\begin{array}{l}{ }^{1} \mathbf{H}-\mathbf{N M R}\left(\mathbf{3 0 0} \mathbf{~ M H z} \mathbf{C D C l}_{3}\right): \delta(\mathrm{ppm}), 1.26-1.34(\mathrm{t}, \mathrm{J}=7.5 \mathrm{~Hz} \text {, } \\
\left.3 \mathrm{H}, \mathrm{CH}_{3}\right), 1.93(\mathrm{~s}, 3 \mathrm{H}, \mathrm{OAc}), 2.04(\mathrm{~s}, 3 \mathrm{H}, \mathrm{OAc}), 2.07(\mathrm{~s}, 3 \mathrm{H}, \mathrm{OAc}), \\
2.09(\mathrm{~s}, 3 \mathrm{H}, \mathrm{OAc}), 2.81-2.88\left(\mathrm{q}, \mathrm{J}=7.2 \mathrm{~Hz}, 2 \mathrm{H}, \mathrm{CH}_{2}\right), 3.98-4.00 \\
(\mathrm{~m}, 1 \mathrm{H}, \mathrm{H}-5), 4.09-4.19(\mathrm{~m}, 1 \mathrm{H}, \mathrm{H}-6 \mathrm{a}), 4.29-4.33(\mathrm{~m}, 1 \mathrm{H}, \mathrm{H}- \\
\text { 6b), } 5.27(\mathrm{t}, \mathrm{J}=9.3 \mathrm{~Hz}, 1 \mathrm{H}, \mathrm{H}-2), 5.42(\mathrm{t}, \mathrm{J}=9.3 \mathrm{~Hz}, 1 \mathrm{H}, \mathrm{H}-4) 5.81 \\
(\mathrm{t}, \mathrm{J}=9.6 \mathrm{~Hz}, 1 \mathrm{H}, \mathrm{H}-3), 6.20(\mathrm{~d}, \mathrm{~J}=9.3 \mathrm{~Hz}, 1 \mathrm{H}, \mathrm{H}-1), 7.48-7.55 \\
(\mathrm{~m}, 3 \mathrm{H}, \mathrm{Ar}), 7.85-7.87(\mathrm{~m}, 2 \mathrm{H}, \mathrm{Ar}), 10.39(\mathrm{~s}, 1 \mathrm{H}, \mathrm{HC}=\mathrm{N})\end{array}$ \\
\hline & $\begin{array}{l}{ }^{13} \mathbf{C}-\mathrm{NMR}\left(\mathbf{7 5} \mathbf{M H z}_{\mathbf{C}} \mathbf{C D C l}_{3}\right): \delta(\mathrm{ppm}), 10.11,18.93,20.73,73.58, \\
\text { 75.32, 77.05, 77.47, 81.46, 81.66, 160.48, 168.77, 169.39, } \\
\text { 170.12, 127.62, 127.75, 128.08, 130.01. Found: C, 53.49; H, } \\
\text { 5.48; N, 9.90; S, 5.60 \%. }\end{array}$ \\
\hline
\end{tabular}

Figure 1) Structural and spectral information of newly synthesized derivatives 
Table 1) Antibacterial activity of the synthesized derivatives (0.5 mg.mL)

\begin{tabular}{|c|c|c|c|c|c|c|c|c|c|c|c|c|}
\hline \multirow{3}{*}{ 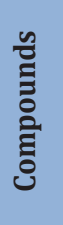 } & \multicolumn{12}{|c|}{ Microorganism } \\
\hline & \multicolumn{3}{|c|}{$\begin{array}{c}\text { E. Coli } \\
\text { PTCC1769 }\end{array}$} & \multicolumn{3}{|c|}{$\begin{array}{l}\text { P. aeruginosa } \\
\text { PTCC1690 }\end{array}$} & \multicolumn{3}{|c|}{$\begin{array}{l}\text { S. aureus } \\
\text { PTCC1917 }\end{array}$} & \multicolumn{3}{|c|}{$\begin{array}{l}\text { B. Cereus } \\
\text { PTCC1015 }\end{array}$} \\
\hline & MBC & MIC & IZ & MBC & MIC & IZ & MBC & MIC & IZ & MBC & MIC & IZ \\
\hline $5 a$ & - & - & - & - & - & - & - & - & $6 \pm 0.7$ & - & - & $6 \pm 0.5$ \\
\hline $7 a$ & 1000 & 1000 & $7 \pm 0.2$ & 1000 & 500 & $7 \pm 0.2$ & - & - & $8 \pm 0.9$ & - & - & $9 \pm 0.8$ \\
\hline $5 b$ & - & - & - & - & - & - & - & - & $6 \pm 0.8$ & - & - & $6 \pm 0.1$ \\
\hline $7 b$ & - & - & $6 \pm 0.3$ & 1000 & 1000 & $7 \pm 0.1$ & 1000 & 1000 & $9 \pm 0.5$ & - & - & $9 \pm 0.1$ \\
\hline $5 c$ & - & - & - & - & - & - & - & - & $8 \pm 0.7$ & - & - & $9 \pm 0.1$ \\
\hline $7 c$ & - & - & $7 \pm 0.8$ & - & - & $6 \pm 0.5$ & 500 & 500 & $14 \pm 0.3$ & 500 & 250 & $16 \pm 0.1$ \\
\hline $5 d$ & - & - & - & - & - & - & - & - & $8 \pm 0.7$ & - & - & $7 \pm 0.9$ \\
\hline $7 d$ & 1000 & 1000 & $7 \pm 0.6$ & - & - & - & 1000 & 500 & $13 \pm 0.1$ & 1000 & 500 & $15 \pm 0.3$ \\
\hline $5 e$ & - & - & - & - & - & - & - & - & $8 \pm 0.1$ & - & - & $7 \pm 0.2$ \\
\hline $7 e$ & - & - & $7 \pm 0.1$ & - & - & - & 1000 & 1000 & $11 \pm 0.1$ & 1000 & 500 & $12 \pm 0.1$ \\
\hline $5 f$ & - & - & NA & - & - & - & - & - & - & - & - & $8 \pm 0.1$ \\
\hline $7 \mathrm{f}$ & - & - & $6 \pm 0.2$ & - & - & $6 \pm 0.5$ & NA & NA & $8 \pm 0.7$ & 1000 & 500 & $11 \pm 0.2$ \\
\hline G & 1000 & 500 & $23 \pm 0.4$ & 500 & 250 & $27 \pm 0.3$ & 250 & 125 & $28 \pm 0.5$ & 125 & 62.50 & $31 \pm 0.6$ \\
\hline
\end{tabular}

IZ: Inhibition Zone.

MIC: Minimum Inhibitory Concentration.

MBC: Minimum Bactericidal Concentration.

7c: 4- (furfuraldehyde amino)-5- etyl- 2-yl- 3- (2, 3, 4, 6-tetra- 0- acetyl- $\beta$ - D-gluco pyranosyl Sulfonyl) -S-triazol. 7d: 4- (benzylidene amino)- 2- yl- 3- (2, 3, 4, 6- tetra- 0- acetyl- $\beta$ - D- gluco pyranosyl Sulfonyl) -S-triazol.

\pm : Averaged three times.

-: No activity.

G: Penicillin G.

aureus, Pseudomonas aeruginosa, and Escherichia coli strains were first opened under sterile conditions and transferred to the nutrient broth (NB) culture medium and incubated at $37{ }^{\circ} \mathrm{C}$ for 24 hours. Also, for fungi samples, ampouls containing Aspergillus niger and Candida albicans strains were first opened under sterile conditions and transferred to the sabouraud dextrose broth (SDB) and incubated at $37^{\circ} \mathrm{C}$ for 24 hours. Using a sampler, $1 \mathrm{~mL}$ of the 24 hour culture of microbial suspension was transferred to a tube containing sterile nutrient broth, and then the turbidity of the microbial suspension was visually compared to the McFarland standard set with a spectrophotometer at $625 \mathrm{~nm}$ and absorption rate of $1.5 \times 10^{9} \mathrm{CFU} / \mathrm{mL}$. For bacterial strains, the nutrient agar culture medium was used for agar well diffusion test, and the nutrient broth culture medium was used to test the dilution in the tubes. For fungal strains, the sabouraud dextrose agar (SDA) culture medium was used for agar well diffusion test, and SDB culture medium was used to test the dilution in the tubes. All environments were prepared according to the manufacturer's instructions and sterilized using an autoclave ${ }^{[24]}$.

Agar well diffusion method: The agar disc diffusion method was used to determine antimicrobial activity of the compounds using a previously described standard method ${ }^{[24]}$.

Broth dilution method: The MIC, MBC and MFC values of the compounds were determined using a previously described standard method ${ }^{[24]}$. 
Table 2) Antifungal activity of the synthesized derivatives (0.5 mg.mL)

\begin{tabular}{|c|c|c|c|c|c|c|}
\hline \multirow{3}{*}{ Compounds } & \multicolumn{6}{|c|}{ Microorganism } \\
\hline & \multicolumn{3}{|c|}{$\begin{array}{l}\text { C. Albicans } \\
\text { PTCC5027 }\end{array}$} & \multicolumn{3}{|c|}{$\begin{array}{c}\text { A. Niger } \\
\text { PTCC5162 }\end{array}$} \\
\hline & MFC & MIC & IZ & MFC & MIC & IZ \\
\hline $5 a$ & - & - & $6 \pm 0.9$ & - & - & $7 \pm 0.1$ \\
\hline $7 a$ & 1000 & 1000 & $12 \pm 0.1$ & 1000 & 1000 & $11 \pm 0.3$ \\
\hline $5 b$ & - & - & $7 \pm 0.2$ & - & - & $7 \pm 0.1$ \\
\hline $7 \mathrm{~b}$ & 1000 & 1000 & $11 \pm 0.3$ & 1000 & 1000 & $11 \pm 0.1$ \\
\hline $5 c$ & - & - & $8 \pm 0.5$ & - & - & $9 \pm 0.1$ \\
\hline $7 \mathrm{c}$ & 250 & 125 & $16 \pm 0.1$ & 250 & 125 & $16 \pm 0.2$ \\
\hline $5 d$ & - & - & $9 \pm 0.1$ & - & - & $8 \pm 0.7$ \\
\hline $7 d$ & 250 & 125 & $18 \pm 0.7$ & 500 & 250 & $14 \pm 0.5$ \\
\hline $5 e$ & - & - & $8 \pm 0.7$ & - & - & $8 \pm 0.1$ \\
\hline $7 e$ & 1000 & 500 & $12 \pm 0.4$ & 1000 & 500 & $12 \pm 0.1$ \\
\hline $5 f$ & - & - & $6 \pm 0.5$ & - & - & $7 \pm 0.3$ \\
\hline $7 f$ & 1000 & 1000 & $12 \pm 0.7$ & 1000 & 1000 & $11 \pm 0.5$ \\
\hline $\mathrm{N}$ & 125 & 62.50 & $26 \pm 0.8$ & 250 & 125 & $22 \pm 0.6$ \\
\hline
\end{tabular}

IZ: Inhibition Zone.

MIC: Minimum Inhibitory Concentration.

MFC: Minimum Fungicidal Concentration.

7c: 4- (furfuraldehyde amino)-5- etyl- 2-yl- 3- (2, 3, 4, 6-tetra- 0- acetyl- $\beta$ - D-gluco pyranosyl Sulfonyl) -S-triazol. 7d: 4- (benzylidene amino)- 2- yl- 3- (2, 3, 4, 6- tetra- 0- acetyl- $\beta$ - D- gluco pyranosyl Sulfonyl) -S-triazol.

\pm : Averaged three times.

-: No activity.

$\mathrm{N}$ : Nystatin.

Statistical methods: All diameter results obtained from three repetitions were reported as mean \pm standard deviation. SPSS statistical software Version 22 was used for data analysis.

\section{Findings}

Chemistry: FT-IR, H-NMR, and C-NMR spectra of all the compounds were obtained (Figure 1). 4-amino-5-alkyl-4HS-triazole-3-thiol 3(a-c) were prepared by condensing aliphatic carboxylic acids (2a-c) with thiocarbohydrazide. This reaction is the selective method for the preparation of 4-amino-5- alkyl-4H-Striazole-3-thiol (Scheme 1). Schiff bases (5a-f) were synthesized by the reaction of 3-alkyl-4-amino-S-triazole-5-thione (3ac) with furan and benzene aldehydes (4de) in absolute ethanol as a solvent in the presence of glacial acetic acid as a catalyst. The ${ }^{1} \mathrm{H}$-NMR spectra showed a singlet signal at about $10 \mathrm{ppm}$ due to $\mathrm{CH}=\mathrm{N}$ and the lack of chemical shift of $4-\mathrm{NH}_{2}$ in the spectra of 3(a-c), proving that the intended Schiff bases (5a-f) were formed. In an attempt to obtain $\alpha$-aceto-bromo-glucose, D-glucose was first treated with acetic anhydride in pyridin at room temperature, resulting in the produciton of $1,2,3,4,6$-penta- $O$-acetyl$\beta$-glucose, then anomeric bromination of this compound with hydrogen bromide in acetic acid resulted in 2,3,4,6-tetra- $O$-acetyl$\alpha$-gluco-pyranosyl-bromide production. The presence of thiol-thione tautomerism is known for the compounds $3(\mathrm{a}-\mathrm{c})$, and generally one form is predominant [25-27]. Thioglycosides of S-triazoles (7a-f) were synthesized by the reaction of 3-alkyl-4- 
amino-S-triazole-5-thione Schiff bases (5a-f) with the peracetylated $\beta$-pyranosyl-bromide ${ }^{[6]}$ in the presence of potassium carbonate as a weak base in dry acetone (Scheme 1). Anomeric $\beta$-configurations of the $S$-linked glycosides (7a-f) were supported by their ${ }^{1} \mathrm{H}-\mathrm{NMR}$ data. The chemical shifts of the anomeric proton signals of thioglycosides were revealed around $\delta(6.20)$ with a large coupling constant $J_{1,2}$ values of $9.3 \mathrm{~Hz}$, consistent with the reported data for S- $\beta-D$ glycosides.

\section{Determination of in Vitro Antimicrobial}

Activity: According to the results, all the compounds had lesser activity than corresponding standard compounds, and the target compounds exhibited better antifungal activity than antibacterial activity. However, the antibacterial activity results showed that compound $7 \mathrm{c}$ with $\mathrm{IZ}=16 \pm 0.1 \mathrm{~mm}$, compound $7 \mathrm{~d}$ with $\mathrm{IZ}=15$ $\pm 0.3 \mathrm{~mm}$, andcompound $7 \mathrm{e}$ with $\mathrm{IZ}=12 \pm$ $0.1 \mathrm{~mm}$ had antibacterial properties against B. Cereus; also, compound $7 \mathrm{c}$ with $\mathrm{IZ}=14$ $\pm 0.3 \mathrm{~mm}$ and compound $7 \mathrm{~d}$ with $\mathrm{IZ}=13$ $\pm 0.1 \mathrm{~mm}$ had antibacterial properties against $S$. aureus. It should be noted that the synthesized compounds did not show antibacterial properties against Gramnegative bacteria (Table 1). The antifungal study results revealed that compound $7 \mathrm{c}$ with $\mathrm{IZ}=16 \pm 0.2 \mathrm{~mm}$ and compound $7 \mathrm{~d}$ with IZ= $14 \pm 0.5 \mathrm{~mm}$ showed antifungal properties against $A$. Niger; also, compound $7 \mathrm{c}$ with $\mathrm{IZ}=16 \pm 0.1 \mathrm{~mm}$ and compound $7 \mathrm{~d}$ with IZ= $18 \pm 0.7 \mathrm{~mm}$ showed antifungal properties against $C$. albicans. It was found that compound $7 \mathrm{c}$ had the highest activity against $B$. Cereus, and compound $7 \mathrm{~d}$ had the highest activity against $C$. albicans among all the tested compounds (Table 2).

\section{Discussion}

The emergence of antibiotic-resistant organisms is a global problem in various societies ${ }^{[1]}$. At present, the response of nosocomial infections to standard antibiotic treatment has changed, and the prevalence of antibiotic resistance in many hospitals has reached dangerous levels ${ }^{[2-3]}$. According to some studies results, about 50 to $60 \%$ of nosocomial infections are caused by antibiotic-resistant strains ${ }^{[28]}$. One of the most important factors contributing to the development of antibiotic resistance is the history of arbitrary use of antibiotics. Antibiotic resistance increases the incidence of complications, mortality, and treatment costs in patients. According to some studies, the per capita consumption of antibiotics has increased significantly in recent years worldwide, which could be due to noncompliance with standard principles of prescribing antibiotics to patients by physicians or could be due to arbitrary consumption of antibiotics by individuals in the community ${ }^{[2]}$. In any case, the mentioned cases have caused the pattern of microbial resistance to change and the prevalence of strains resistant to the first line and even the second line treatment to increase. In the meantime, there is a need to introduce and explore new drug structures that could be used in the synthesis of various drug formulations ${ }^{[29]}$. In this study, novel structures of S-triazoles (7a-f) were synthesized, and their antimicrobial properties were investigated. According to the antibacterial activity results of the compounds, compounds $7 \mathrm{c}$ and $7 \mathrm{~d}$ had an acceptable effect on Gram-positive bacteria. It was thought that the presence of these compounds along with the structure of triazole increased the antibacterial properties of the derivatives. In this regard, El Ashry et al. (2021) synthesized some new derivatives of 5- phenyl- 2, 4-dihydro- $3 \mathrm{H}$ S-triazole as a combination with V-triazole and studied their antibacterial properties, the antibacterial activity of some compounds was the highest against $E$. coli and $S$. aureus [30]. In the present study, the greatest effect of the compounds was against $C$. albicans, so that both $7 \mathrm{c}$ and $7 \mathrm{~d}$ compounds exhibited significant effects. In a study by 
Revanasiddappa et al. (2017), the best effect of these compounds was reported to be against $A$. fumigatus ${ }^{[31]}$, which is consistent with the present study result. Godeau et al. (2021) stated that Difenoconazole is a triazole fungicide widely used in agriculture to protect various crops [32]. According to the results of this study and similar studies, triazole-based compounds could be considered as a good candidate for use in the development of common antifungal drugs that are commonly used today.

\section{Conclusion}

A series of new thioglycoside derivatives of S-triazoles (7a-f) were synthesized. The triazoles (3a-c) were produced via the reaction of thiocarbohydrazide with aliphatic carboxylic acids. The Schiff bases (5a-f) were produced through the reaction of 4-amino-5-alkyl-S-triazole-3-thione with appropriate aryl aldehydes (furan and benzne) and then heated under reflux in ethanol and in the presence of glacial acetic acid. S-glycosilytion of Schiff bases was performed by aceto bromo glucose in acetone in the presence of potassium carbonate. The structures of the target compounds were characterized by ${ }^{1} \mathrm{H}$ NMR, ${ }^{13} \mathrm{C}$ NMR, and FTIR. All the newly synthesized compounds were evaluated for their antimicrobial activities in vitro against two Gram-positive bacteria, two Gram-negative bacteria, and two fungi. These compounds exhibited better antifungal activity than antibacterial activity. Though compound $7 \mathrm{c}$ was found to have the highest activity against $B$. Cereus, and compound $7 \mathrm{~d}$ was found to have the highest activity against $C$. albicans among all the tested compounds.

\section{Supplementary materials}

$(5 a)=4$ - ((furan- 2- yl- methylen) amino)2, 4-dihydro- 3H- S-triazol- 3-thion: (Light Yellow crystals) (Yield\% $=75)$ (0.52 g) m.p: 173 - $175^{\circ} \mathrm{C}$. FT-IR (KBr, v cm${ }^{-1}$ ): 3108, 3068, $2995(\mathrm{~N}-\mathrm{H}), 1546(\mathrm{C}=\mathrm{N}), 1480(\mathrm{C}=\mathrm{S}), 1275$ (C-C). ${ }^{1} \mathrm{H}-\mathrm{NMR}$ (300 MHz, DMSO): $\delta$ (ppm), $6.72(\mathrm{~s}, 1 \mathrm{H}$, Furyl), $7.30-7.31(\mathrm{~d}, \mathrm{~J}=3 \mathrm{~Hz}$, 1H, Furyl), 8.20 (s, 1H, Furyl), 8.41 (s, 1H, C$\mathrm{H}), 9.78(\mathrm{~s}, 1 \mathrm{H}, \mathrm{HC}=\mathrm{N}), 13.64(\mathrm{~s}, 1 \mathrm{H}, \mathrm{N}-\mathrm{H})$. ${ }^{13} \mathrm{C}$-NMR (75 $\mathrm{MHz}, \mathrm{CDCl}_{3}$ ): Found: $\mathrm{C}, 43.15$; $\mathrm{H}, 3.39$; N, 28.60; S, 16.63 .

$(5 b)=4$ - ((furan- 2-yl-methylene) amino)- 5methyl- 2, 4-dihydro- 3H- S-triazol- 3-thion: (Light Yellow crystals) (Yield\% $=77)(0.80$ g), m.p: $167-169^{\circ} \mathrm{C}$. FT-IR (KBr, u cm$\left.{ }^{-1}\right)$ : 3118, $2940(\mathrm{~N}-\mathrm{H}), 1600(\mathrm{C}=\mathrm{N}), 1488(\mathrm{C}=$ S), 1266 (C-C), ${ }^{1} \mathrm{H}-\mathrm{NMR}(300 \mathrm{MHz}, \mathrm{DMSO}): \delta$ (ppm), 2.28 (s, 3H, CH3), 6.74 (s, 1H, Furyl), 7.29 - 7.30 (d, J=3 Hz, 1H, Furyl), 8.00 (s, 1H, Furyl), 9.75 (s, 1H, HC= N), $13.75(\mathrm{~s}, 1 \mathrm{H}$, $\mathrm{N}-\mathrm{H}$ ). ${ }^{13} \mathrm{C}-\mathrm{NMR}$ (75 MHz, $\mathrm{CDCl}_{3}$ ): Found: C, 45.19; H, 3.95; N, 24.99; S, 15.32.

$(5 c)=5$ - ethyl- 4- ((furan- 2- yl- methylene) amino)-2 ,4- dihydro- 3H- S-triazol- 3-thion: (Light Brown crystals), (Yield\% $\%$ 86) (0.6g), m.p: 177 - $179^{\circ} \mathrm{C}$. FT-IR (KBr, v cm$\left.{ }^{-1}\right)$ : 3112 , 3076, $2945(\mathrm{~N}-\mathrm{H}), 1594(\mathrm{C}=\mathrm{N}), 1484(\mathrm{C}=$ S), 1280 (C- C). ${ }^{1} \mathrm{H}-\mathrm{NMR}$ (300 MHz, DMSO): $\delta(\mathrm{ppm}), \delta 1.19$ (t, J=7.5 Hz, 3H, CH3), 2.68 (q, J=7.5 Hz, 2H, CH2), 6.75 (s, 1H, Furyl), 7.31 (d, J=3.3 Hz, 1H, Furyl), 8.04 (s, 1H, Furyl), 9.87 (s, 1H, HC= N), $13.73(\mathrm{~s}, 1 \mathrm{H}, \mathrm{NH})$. ${ }^{13} \mathrm{C}-\mathrm{NMR}\left(75 \mathrm{MHz}, \mathrm{CDCl}_{3}\right.$ ): Found: C, 48.58; H, 4.40; N, 25.41; S, 14.60 .

$(5 d)=4$ - (benzylidene- amino)- 2, 4-dihydro3H- S-triazol- 3-thion: (White crystals), (Yield\% = 74) (0.5 g), m.p: $158-160{ }^{\circ} \mathrm{C}$, FT-IR (KBr, v cm$\left.{ }^{-1}\right)$ : 3104, 3073, $3000(\mathrm{~N}-$ $\mathrm{H}), 2870(\mathrm{C}-\mathrm{H}), 1552(\mathrm{C}=\mathrm{N}), 1488(\mathrm{C}=\mathrm{S})$. ${ }^{1} \mathrm{H}-\mathrm{NMR}$ (300 MHz, DMSO): $\delta$ (ppm), 7.53 7.57 (m, 3H, Ar), $7.86-7.88$ (m, 2H, Ar), 9.85 $(\mathrm{s}, 1 \mathrm{H}, \mathrm{HC}=\mathrm{N}) 9.83(\mathrm{~s}, 1 \mathrm{H}, \mathrm{HC}=\mathrm{N}), 13.58(\mathrm{~s}$, $1 \mathrm{H}, \mathrm{NH}) .{ }^{13} \mathrm{C}-\mathrm{NMR}\left(75 \mathrm{MHz}, \mathrm{CDCl}_{3}\right.$ ): Found: $\mathrm{C}$, 52.79; H, 3.90; N, 27.62; S, 15.81 .

$(5 \mathrm{e})=4$ - (benzylidene- amino)- 5- methyl2, 4-dihydro- 3H- S-triazol- 3-thion: (Light Yellow crystals), (Yield\% $=85$ ) (0.8 g), m.p: $188-190^{\circ} \mathrm{C}$, FT-IR (KBr, v cm${ }^{-1}$ ): 3066, 2930 $(\mathrm{N}-\mathrm{H}), 1584(\mathrm{C}=\mathrm{N}), 1493(\mathrm{C}=\mathrm{S}) \cdot{ }^{1} \mathrm{H}-\mathrm{NMR}$ (300 MHz, DMSO): $\delta(\mathrm{ppm}), 2.32(\mathrm{~s}, 3 \mathrm{H}$, CH3), 7.53 - 7.57 (m, 3H, Ar), $7.86-7.88$ (m, 2H, Ar), $9.85(\mathrm{~s}, 1 \mathrm{H}, \mathrm{HC}=\mathrm{N}), 13.65(\mathrm{~s}, 1 \mathrm{H}$, $\mathrm{NH}) \cdot{ }^{13} \mathrm{C}-\mathrm{NMR}\left(75 \mathrm{MHz}, \mathrm{CDCl}_{3}\right.$ ): Found: C, 
52.79; H, 3.90; N, 27.62; S, 15.81.

(5f) = 4- (benzylidene- amino)- 5- ethyl- 2, 4-dihydro- 3H- S-triazol- 3-thion: (White crystals), (Yield\% $\%$ 76) (0.5 g), m.p:160$162^{\circ} \mathrm{C}$, FT-IR $\left(\mathrm{KBr}, \mathrm{v} \mathrm{cm}{ }^{-1}\right): 3100,3060,2934$ $(\mathrm{NH}), 1577(\mathrm{C}=\mathrm{N}), 1493(\mathrm{C}=\mathrm{S}) \cdot{ }^{1} \mathrm{H}-\mathrm{NMR}$ (300 MHz, DMSO): $\delta(\mathrm{ppm}), 1.17-1.22(\mathrm{t}$, $\mathrm{J}=7.5 \mathrm{~Hz}, 3 \mathrm{H}, \mathrm{CH} 3), 2.67-2.75(\mathrm{q}, \mathrm{J}=7.5 \mathrm{~Hz}$, 2H, CH2), 7.54 - 7.61 (m, 3H, Ar), 7.86 - 7.89 (m, 2H, Ar), 9.86 (s, 1H, CH), 13.69 (s, 1H, $\mathrm{NH}) \cdot{ }^{13} \mathrm{C}-\mathrm{NMR}\left(75 \mathrm{MHz}, \mathrm{CDCl}_{3}\right.$ ): Found: C, 56.93; H, 5.14; N, 24.35; S, 13.70.

Synthesis of 1, 2, 3, 4, 6-penta-0-acetyl$\beta$-D-glucopyranose: (White precipitate), (Yield $\%=68)(7.5 \mathrm{~g})$, FT-IR (KBr, $\left.v \mathrm{~cm}^{-1}\right)$ : 1748, 1374, 1227, ${ }^{1} \mathrm{H}-\mathrm{NMR}(300 \mathrm{MHz}$, CDCl3): $\delta$ (ppm), 2.01 (s, 3H, OAc), 2.03 (s, 3H, OAc), 2.08 (s, 3H, OAc), $2.11(\mathrm{~s}, 3 \mathrm{H}$, OAc), $2.18(\mathrm{~s}, 3 \mathrm{H}, \mathrm{OAc}), 3.82-3.85(\mathrm{~m}, 1 \mathrm{H}$, H-5), 4.08 - 4.12 (m, 1H, H- 6a), $4.26-4.32$ (m, 1H, H- 6b), $5.09-5.28$ (m, 3H, H- 2, H- 4, H- 3), 5.71 (d, 1H, J1, $2=8.4, \mathrm{H}-1$ ). ${ }^{13} \mathrm{C}$-NMR (75 MHz, CDCl3): $\delta$ (ppm), 20.55 (3C), 20.69, 20.80 (50COCH3), 61.41 (C6), 67.70 (C- 4), 70.18 (C- 2), 72.69 (C- 3), 72.75 (C- 5), 91.66 (C- 1), 168.94, 169.23, 169.37, 170.08, 170.59 (5 OCOCH3). Calcd: C, 52.58; H, 6.23 \%; Found: C, 52.68; H, 6.11; $0 \%$.

Synthesis of 2, 3, 4, 6-tetra- 0- acetyl$\alpha$-D-glucopyranosyl bromide: (White precipitate),( Yield $\%=57)$ (1.2 g), FTIR (KBr, $\left.v \mathrm{~cm}^{-1}\right): 1745,1377,1236,607$, ${ }^{1} \mathrm{H}-\mathrm{NMR}$ (300 MHz, CDCl3): $\delta$ (ppm), 2.03 (s, 3H, OAc), 2.05 (s, 3H, OAc), $2.10(\mathrm{~s}, 6 \mathrm{H}$, OAc), 4.12 (d, 1H, H-6a), $4.28-4.36(\mathrm{~m}, 2 \mathrm{H}$, H-6b, H-5), 4.81 - 4.86 (dd, 1H, J1,2 = 3.9, $\mathrm{J} 2,3=9.9, \mathrm{H}-2), 5.16(\mathrm{t}, 1 \mathrm{H}, \mathrm{J}=9.9, \mathrm{H}-4), 5.56$ $(\mathrm{t}, 1 \mathrm{H}, \mathrm{J}=9.9, \mathrm{H}-3), 6.61(\mathrm{~d}, 1 \mathrm{H}, \mathrm{J} 1,2=3.9$, $\mathrm{H}-1) .{ }^{13} \mathrm{C}-\mathrm{NMR}(75 \mathrm{MHz}, \mathrm{CDCl} 3): \delta$ (ppm), 20.53, 20.60 (2C), 20.63 (40COCH3), 60.93 (C- 6), 67.15 (C- 4), 70.14 (C- 2), 70.58 (C3), 72.12 (C- 5), $86.54\left(\mathrm{C}^{-1}\right.$ ), 169.44, 169.77, 169.82, 170.48 (40COCH3). Calcd: C, 44.03; H, $5.17 \%$; Found: C, 43.19; H, $5.09 \%$.

Acknowledgments: The authors are grateful to Urmia University for providing a fellowship for the present work.

Ethical Permission: This work was approved by the Ethics Committee of Urmia University, Urmia, Iran.

Conflicts of Interest: There is no conflict of interest.

Authors' contribution: Conceptualization: KA,ZD; Data curation and formal analysis: KA,ZD,YS,KA; Investigation: KA,ZD,YS,KA; Methodology and project administration: KA,ZD,YS; Supervision: KA,ZD; Validation: KA,ZD,YS; Writing of original draft: KA,YS; Writing, reviewing, and editing: KA,YS.

Fundings: This research was the R.F master's thesis (Code: 2-3039), done in Department of Organic Chemistry, Faculty of Chemistry, Urmia University, Urmia, Iran.

Consent to participate: There was no need.

\section{References}

1. Willyard C. The drug-resistant bacteria that pose the greatest health threats. Nature. 2017;543(7643):15.

2. Morris S, Cerceo E. Trends, epidemiology, and management of multi-drug resistant gram-negative bacterial infections in the hospitalized setting. Antibiotics. 2020 Apr;9(4):196.

3. Sultan I, Rahman S, Jan AT, Siddiqui MT, Mondal AH, Haq QM. Antibiotics, resistome, and resistance mechanisms: A bacterial perspective. Front Microbiol. 2018;9:2066.

4. Maddila S, Momin $\mathrm{M}$, Gorle $\mathrm{S}$, Palakondu L, Jonnalagadda SB. Synthesis and antioxidant evaluation of novel phenothiazine linked substitutedbenzylideneamino-1, 2, 4-triazole derivatives. J Chil Chem Soc. $2015 ; 60$ (2):2919-23.

5. Sangshetti JN, Lokwani DK, Sarkate AP, Shinde DB. Synthesis, antifungal activity, and docking study of some new 1, 2, 4triazole analogs. Chem Biol Drug Des. 2011 Nov;78(5):800-9.

6. Ragenoviќ KC, Dimova V, Kakurinov V, Gabor DM, Buzarovska A. Synthesis and 
antibacterial, and antifungal activity of 4-substituted-5-aryl-1, 2, 4-triazole. Molecules. 2001;6(10):815-24.

7. MathewV, Keshavayya J, Vaidya VP, Giles D. Studies on synthesis and pharmacological activities of 3, 6-disubstituted-1, 2, 4-triazolo [3, 4-b]-1, 3, 4-thiadiazoles and their dihydro analogues. Eur J Med Chem. 2007;42(6):823-40.

8. Altıntop MD, Kaplancıklı ZA, TuranZitouni G, Özdemir A, İșcan G, Akalın G, et al. Synthsis and anticandidal activity of new triazolothiadiazine derivatives. Eur J Med Chem. 2011;46(11):5562-6.

9. Mathew V, Giles D, Keshavayya J, Vaidya VP. Studies on synthesis and pharmacological activities of 1, 2, 4triazolo [3, 4-b] 1, 3, 4-thiadiazoles and their dihydro analogues. Arch Pharm. 2009;342(4):210-22.

10. Ahmadi F, Rezayan Ghayahbashi M, Sharifzadeh M, Alipoiur E, Nasser Ostad $\mathrm{S}$, Vosooghi $\mathrm{M}$, et al. Synthesis and evaluation of anti-inflammatory and analgesic activities of new 1, 2, 4-triazole derivatives. Med Chem. 2015;11(1):6976.

11. Kaur R, Ranjan Dwivedi A, Kumar B, Kumar V. Recent developments on 1, 2, 4-triazole nucleus in anticancer compounds: A review. Anticancer Agents Med Chem. 2016;16(4):465-89.

12. Ronad PM, Noolvi MN, Sapkal S, Dharbhamulla S, Maddi VS. Synthesis and antimicrobial activity of 7-(2-substituted phenylthiazolidinyl)-benzopyran2-one derivatives. Eur J Med Chem. 2010;45(1):85-9.

13. Dilmaghani KA, Jazani NH. Nasuhi Pur F, Shokoufeh N, Ghadiri F, Mirfakhraee F. Synthesis of 5-(4-aminophenyl)-2(arylamino)-1, 3, 4-thiadiazoles and their schiff base derivatives as antimycobacterial agents. Chem Heterocycl Compd. 2012;48:362-7.

14. Gupta D, Jain DK. Synthesis, antifungal, and antibacterial activity of novel 1 ,
2, 4-triazole derivatives. J Adv Pharm Technol Res. 2015;6(3):141-6.

15. Dilmaghani KA, Nasuhi Pur F, Hataminezhad M. Synthesis and antibacterial evaluation of new thione substituted 1, 2, 4-triazole Schiff bases as novel antimicrobial agents. Iran J Pharm Res. 2015;14(3):693-9.

16. Dhapalapur MG, Sabnis SS, Deliwala CV. Potential anticancer agents. II. Schiff bases from benzaldehyde nitrogen mustards. J Med Chem. 1968;11(5):1014-9.

17. Khalil NS. Efficient synthesis, structure, and antimicrobial activity of some novel $\mathrm{N}$-and $\mathrm{S}-\beta$-D-glucosides of 5-pyridin3-yl-1, 2, 4-triazoles. Carbohydr Res. 2006;341(13):2187-99.

18. Tiwari J, Saquib M, Singh S, Tufail F, Singh J, Singh J. Catalyst-free glycerol-mediated green synthesis of $5^{\prime}$-thioxospiro [indoline-3, 3'-[1, 2, 4] triazolidin]-2-ones/spiro [indoline-3, $3^{\prime}$ [1, 2, 4] triazolidine]-2, 5'-diones. Synth Commun. 2017;47(21):1999-2006.

19. Perrin DD, Armarego WL, Perrin DR. Purification of laboratory chemicals pergamon. New York; 1988.

20. BeyerH,KrogerCF,BusselG.1,2,4-Triazoles. I. The reaction of thiocarbohydrazide and thiosemicarbazide with aliphatic carbonic acids and their derivatives. Ann. 1960;637:135-45.

21. de Mora SJ, Fowler SW, Cassi R, Tolosa I. Assessment of organotin contamination in marine sediments and biota from the Gulf and adjacent region. Mar Pollut Bull. 2003;46(4):401-9.

22. Furniss BS. Text book of practical organic chemistry. 1989.

23. Dilmaghani KA, Pur FN, Jazani NH, Alavi A, Niknam Z, Mirfakhraee F. Synthesis of new 1, 2, 4-triazole-5-thiones and their thioglycoside derivatives as potential antibacterial agents. Phosphorus Sulfur Silicon Relat Elem. 2014;189(1):81-7.

24. Zarrabi N, souldozi A, SarveAhrabi Y. In vitro evaluation of antibacterial and 
antifungal properties of some new 1, 3, 4-oxadiazole derivatives containing phenyl group. IEM. 2020;6(3):177-92

25. Senda Y, Imaizumi S. Carbon-13 pulse Fourier transform NMR: Conformational preference of the hydroxyl and the acetoxyl group in 2-cyclohexenol and its acetate. Tetrahedron. 1974;30(21):3813-5.

26. Kelarev VI, Shvekhgeimer GA, Lunin AF. Synthesis of 2-amino-1, 3, 4-oxadiazoles, 2-amino-1, 3, 4-thiadiazoles, and 1, 2, 4-triazoline-3-thiones containing indolyl radicals. Chem Heterocycl Com. 1984;20(9):1043-8.

27. Gopinathan S, Pardhy SA, Budhkar AP, Gopinathan C. Ruthenium (II) complexes of nitrogen and sulphur containing heterocyclic ligands. Synth React Inorg Met Org Chem. 1988;18(8):823-36.

28. Ghadiri H, Vaez H, Khosravi S, Soleymani E. The antibiotic resistance profiles of bacterial strains isolated from patients with hospitalacquired bloodstream and urinary tract infections. Crit Care Res Pract. 2012;2012.
29. Micoli F, Bagnoli F, Rappuoli R, Serruto D. The role of vaccines in combatting antimicrobial resistance. Natr Rev Microbiol. 2021;19:287-302.

30. El Ashry ES, Elshatanofy MM, Badawy ME, Kandeel KM, Elhady OM, Abdel-Sayed MA. Synthesis and evaluation of antioxidant, antibacterial, and target proteinmolecular docking of novel 5-phenyl-2, 4-dihydro-3 H-1, 2, 4-triazole derivatives hybridized with 1, 2, 3-triazole via the flexible SCH 2-bonding. Russ J Gen Chem. 2020;90(12):2419-34.

31. Revanasiddappa BC, Kumar MV, Nayak P, Ali AR, Jisha MS. Synthesis, antibacterial, and antifungal evaluation of novel pyrazoline derivatives. Res J Pharm Tech. 2017;10(5):1481.

32. Godeau C, Morin-Crini N, Staelens JN, Martel B, Rocchi S, Chanet G, et al. Adsorption of a triazole antifungal agent, difenoconazole, on soils from a cereal farm: Protective effect of hemp felt. Environ Technol Inno. 2021;22:101394. 